\title{
Malignancy in chronic osteomyelitis of the foot and ankle (case series)
}

\author{
N.M. Kliushin, A.S. Sudnitsyn, N.S. Migalkin, T.A. Stupina, N.R. Suvorov \\ Russian Ilizarov Scientific Center for Restorative Traumatology and Orthopaedics, Kurgan, Russian Federation
}

\begin{abstract}
Introduction Carcinomatous degeneration is a severe complication of chronic osteomyelitis. The exact mechanism of the malignant transformation remains unknown. This degeneration most often results from squamous cell carcinoma arising in chronic osteomyelitis. Early diagnosis and appropriate treatment are essential for prognosis and final results in carcinomatous degeneration secondary to chronic osteomyelitis. Definitive treatment of chronic osteomyelitis and antimicrobial therapy are thought to be effective in prevention of malignant changes. Objective To identify diagnostic criteria for neoplastic disease in chronic osteomyelitis. Methods We reviewed 7 (2.75\%) patients aged 39 to 65 years who were treated for relapse of bone infection at the Clinic of Osteology Infection between 2017 and 2018. The average duration of the disease was $36 \pm$ 6.42 years. A comprehensive examination performed for the patients on admission included radiographs of the involved limb segment, microbiological analysis of wound discharge, complete blood count, serum biochemistry panels, Doppler ultrasonography of the lower limb arteries and an excisional biopsy. Results Squamous cell carcinoma was diagnosed in the feet of all patients. Discussion The examinations performed were useful for identifying several diagnostic criteria for neoplastic process associated with pseudocarcinomatous hyperplasia in chronic osteomyelitis.

Keywords: chronic osteomyelitis in the foot, malignancy, squamous cell carcinoma, diagnosis of squamous cell carcinoma
\end{abstract}

\section{INTRODUCTION}

Carcinomatous degeneration is a severe complication of chronic osteomyelitis [1-5]. Neoplasms as a result of chronic insults were described as early as during the Roman Empire. Aulus Cornelius Celsus, the Roman author, reported neoplasms from infectious causes in his systematic treatise on medicine [6]. In the mid-19th century Hawkins and Marjolin correlated these skin diseases with osteomyelitis. Mayo Clinic study reported about 4000 cases of chronic osteomyelitis, and malignant lesions were noted in $23 \%$ of the patients [7]. Squamous cell carcinoma is the most common type of malignant tumor resulting from chronic osteomyelitis. The exact mechanism of the malignant transformation remains unknown and is assumed multifactorial. Inflammation, especially chronic one, is the definite cause for tumor development and progression, and it is well referred to as inflammation-related carcinogenesis. In these conditions, the immune system is dysregulated: inflammatory mediators and cytokines expressed by the immune system modulate the genic expression of various proteins, including p53. Furthermore, avascular areas and lymphatic duct obliteration are conditions that discourage antigen presentation. Polymicrobial infection sites are also characterized by horizontal gene transfer and consequent latent mutations that interfere with the immune response [2]. There is evidence that carcinomatous transformation can follow a shift in bacterial flora. Gram-positive bacteria can be replaced by predominant gram-negative flora that produce endotoxins associated with cancer [8]. The duration of osteomyelitis appears to be the principal factor related to carcinogenesis onset, with a minimum latency period of 20 years or more [4].

Early recognition and timely therapy of malignant transformation are essential for prognosis and survival rate reducing the morbidity and mortality associated with human cancers. Debridement and antibacterial therapy are the most effective methods of preventing the onset of these malignancies being the appropriate and definitive treatment of chronic osteomyelitis $[5,9]$.

Radical amputation surgery is accepted as the best treatment option for carcinomatous transformations of chronic bone infections treating both the tumoral and infectious processes. Avoiding amputation, wide resections can result in local recurrence, which can require delayed amputation with worse outcome. Amputation allows a faster rehabilitation and safer recovery avoiding recurrence of neoplasm

Kliushin N.M., Sudnitsyn A.S., Migalkin N.S., Stupina T.A., Suvorov N.R. Malignancy in chronic osteomyelitis of the foot and ankle (case series). Genij Ortopedii, 2019, vol. 25, no 4, pp. 517-522. DOI 10.18019/1028-4427-2019-25-4-517-522. (In Russian) 
and chronic osteomyelitis. It is a tough choice that involves both physical and psychological issues, but it can guarantee better quality of life. The prognosis is generally better for well differentiated squamous cell carcinoma than for other differentiated histological types. The existence of lymphatic node involvement or visceral metastasis lowers the five-year survival rate to $35-50 \%$ [10]. Patients who have no signs of lymph node metastases within 3 years with the tumor properly excised have a good prognosis [11].

Objective Our purpose was to identify diagnostic criteria for neoplastic disease in chronic osteomyelitis.

\section{MATERIAL AND METHODS}

We reviewed 7 (2.75\%) patients aged 39 to 65 years who were treated for relapse of bone infection at the Clinic of Osteology Infection between 2017 and 2018. All patients were diagnosed with squamous cell carcinoma. The patients were males aged from 39 to 65 years (mean age, $52.6 \pm 8.33$ years). The patients sustained an injury to the foot 25 to 44 years ago (on average, $36 \pm 6.42$ years) that complicated with osteomyelitis. The foot injury was accompanied by an injury to the femoral artery in two cases. The patients reported recurrent exacerbations of purulent inflammation treated by courses of antimicrobial therapy and local management. However, the patients developed exacerbations of purulent inflammation from 1 year to 6 years (on average, $3 \pm 1.82$ years) prior to admission to the clinic. The study was performed in accordance with ethical principles for medical research involving human subjects stated in the Declaration of Helsinki developed by the World Medical Association as revised in 2013. Written informed consent was obtained from all patients for publication of the findings without identifying details.

A comprehensive examination performed for the patients on admission included radiographs of the involved limb segment, microbiological analysis of wound discharge, complete blood count, serum biochemistry panels, Doppler ultrasonography of the lower limb arteries and an excisional biopsy.

Preoperative radiological examination was performed for all patients using RADIOTEX radiography system, registration certificate FS № 2006/527; RAYMAT ASI, registration certificate FS № 2006/2099; CLINOMAT, registration certificate FS № 2006/559. Preoperative ultrasound was produced with AVISUS Hitachi ultrasound scanning system (Japan) and 7.5 MHz linear transducer. Duplex scanning provided images of vessels, measurement of blood flow velocity and spectrum analysis. An area of metatarsal metaphysis was referred to as a control ( $\mathrm{AP}=210 \pm 10 \mathrm{CU}$ ).

Intraoperative wound sampling was performed for microbiological studies. Nutrient agar containing $5 \%$ of blood, egg-yolk salt agar, Levine medium, Sabouraud agar growth medium were used to cultivate aerobic and facultative anaerobic bacteria. Cultures grew at $37^{\circ} \mathrm{C}$ for $24-48$ hours. Bacterial counts in clinical isolates were measured using solid agar culture media in accordance with procedural guidelines using decimal logarithm of evolved colonies $(\mathrm{CFC} / \mathrm{mL})$. Bacterial cultures were generically and specifically identified with conventional techniques to explore their tinctorial, cultural and biochemical characteristics, and ATB Expression Bacteriology analyzers (Bio Merieux, France), Walk Away 40 Automated Microbiology Assay System (USA) and WHONET 5.6 Microbiology laboratory database software. Involved bone fragments, sequesters and the soft tissues excised during surgical debridement were histologically examined. Specimen were fixed in $10 \%$ neutral buffered formalin, decalcified in a mixed solution of saline and formic acids, dehydrated in alcohol (70 \% and absolute alcohol), impregnated and embedded in paraffin. Histological preparations (paraffin sections of $4-5 \mathrm{mcm}$ cut with Reichard sledge microtome (Germany) were stained with hematoxylin and eosin and the Masson's trichrome. Images of micropreparations were acquired with AxioCam digital camera, AxioScope.A1 microscope and Zen blue software (Carl Zeiss MicroImaging $\mathrm{GmbH}$, Germany).

\section{RESULTS}

Physical examination showed skin hyperemia of the involved limb, discharging wounds with malodor and abundant serohemorrhagic exudate. The wound bottom was lined with loose fibrinous granulations that could be easily injured having elevated catershaped edges. The wounds were localized on the lateral $(n=2)$, supportive $(n=4)$ and posterior $(n=1)$ surface of the foot and the ankle. The wound 
area measured from 20 to $266 \mathrm{~cm}^{2}$, and averaged to $143.3 \pm 99.4 \mathrm{~cm}^{2}$. Scars were observed close to the purulent nidus in all cases. No pulse could be palpated in the major arteries of the involved limb. Doppler ultrasonography revealed stenosis at the level of the femoral artery $(n=2)$ and weak arterial blood flow in lower limbs $(n=5)$. The patients could ambulate with additional means of support bearing no weight on the involved limb. Functionality of lower limbs was measured with AOFAS and averaged to $59 \pm 2.72$ points.

Radiographs of the involved limb showed bone deformity in patients with evident signs of disarranged bone structure and density. Signs of osteoporosis, resorption and periosteal reaction were seen in all patients. Bone defects were observed somewhere and in the whole bone $(n=6)$. The bone appeared to be sclerotic in 5 cases. Arthrosis $(n=4)$ and ankylosis $(n=2)$ were seen in the ankle joint. Unconfined radiopaque clusters were seen in the soft tissues $(n=6)$. Soft tissue samples were taken from different sites (lateral edge, the core, distal edge) of the wound bottom for microbial analysis. There were total 10 pathogens identified (Table 1) with gram-negative bacteria being most common (70\%). Pseudomonas species were detected in $20 \%$ of the cases. Grampositive bacteria (30\%) included Streptococcus and Staphylococcus species.

Table 1

Types of pathogens detected in the patients

\begin{tabular}{|l|c|c|}
\hline Type of pathogen & $\begin{array}{c}\mathrm{CFU} / \\
\mathrm{mL}\end{array}$ & $\begin{array}{c}\text { Number of } \\
\text { observations }\end{array}$ \\
\hline Pseudomonas aeruginosa & $10^{6}$ & 3 \\
\hline Pseudomonas fluorescens & $10^{6}$ & 1 \\
\hline Streptococcus sp b hemolitico & $10^{6}$ & 1 \\
\hline $\begin{array}{l}\text { Staphylococcus saprophyticus } \\
\text { MRSS }\end{array}$ & $10^{5}$ & 1 \\
\hline Enterococcus faecalis & $10^{4}$ & 2 \\
\hline Carobacter koseri & $10^{5}$ & 1 \\
\hline Staphylococcus warneri & $10^{6}$ & 1 \\
\hline Proteus vulgaris & $10^{3}$ & 1 \\
\hline Providencia rettgeri & $10^{3}$ & 1 \\
\hline Chryseobacterium indologenes & $10^{3}$ & 1 \\
\hline
\end{tabular}

Common blood count and biochemistry panel revealed mild anemia, mild leukocytosis and elevated ESR level in all patients (Table 2). Leukocyte formula showed normal values. Total protein values were normal in the group of patients with elevated CRP and low serum albumin level.

Table 2

Measurements of common blood count and biochemistry panel

\begin{tabular}{|l|c|c|}
\hline $\begin{array}{c}\text { Parameter } \\
\text { (measurement unit) }\end{array}$ & Average value & $\begin{array}{c}\text { Normal } \\
\text { reference values }\end{array}$ \\
\hline \multicolumn{3}{|c|}{ CBC } \\
\hline RBC $\left(10^{12} / \mathrm{L}\right)$ & $4.076 \pm 0.407$ & $4-6$ \\
\hline Hemoglobulin (g/L) & $116.43 \pm 25.150$ & $130-160$ \\
\hline WBC (10\%/L) & $10.41 \pm 2.222$ & $4-9$ \\
\hline ESR (mm/hour) & $93.86 \pm 22.203$ & $0-20$ \\
\hline \multicolumn{3}{|c|}{ Leukocyte formula } \\
\hline Stab neutrophils (\%) & $5.71 \pm 2.603$ & $1-6$ \\
\hline Granulocytes (\%) & $63 \pm 4.342$ & $47-72$ \\
\hline Eosinophils (\%) & $2.03 \pm 0.969$ & $0-5$ \\
\hline Monocytes (\%) & $4.26 \pm 1.866$ & $3-11$ \\
\hline Lymphocytes (\%) & $24.87 \pm 6.321$ & $19-45$ \\
\hline \multicolumn{3}{|c|}{ Biochemistry panel } \\
\hline Total protein (g/L) & $67.9 \pm 5.956$ & $64-83$ \\
\hline $\begin{array}{l}\text { C-reactive protein } \\
\text { (mg/L) }\end{array}$ & $61.8 \pm 23.862$ & $0-5$ \\
\hline Albumin (g/L) & $32.34 \pm 4.422$ & $34-48$ \\
\hline
\end{tabular}

Excisional biopsy was produced for the patients who exhibited clinical sings being not typical for chronic osteomyelitis. Pathohistological evaluation showed bands in the basal and the spinous layers of the epidermis permeating through the deeper dermal layers (Fig. 1).

Normal and atypical cells characterized by altered shape, increase or decrease in size, pathological mitoses were identified (Fig. 2). Singular mitoses were seen per field of view in 6 patients, and one patient showed 2 to 5 mitoses per FOV. Focal diffuse lymphohistiocytic infiltration was evident (Fig. 3), and clusters of pseudomycelium structures of mycotic bacteria seen in one observation (Fig. 3b).

All the samples exhibited corneous pearls (Fig. 4) with less differentiated, rounded cells having a narrow cytoplastic rim and hyperchromic nuclei. Corneous masses were seen as purple concentric formations in the center of the arrangement in evident keratosis and were suggestive of malignancy. On the whole, changes in osseous structures were consistent with chronic osteomyelitis, and neoplastic epithelial patterns invaded through the intertrabecular spaces (Fig. 5). 

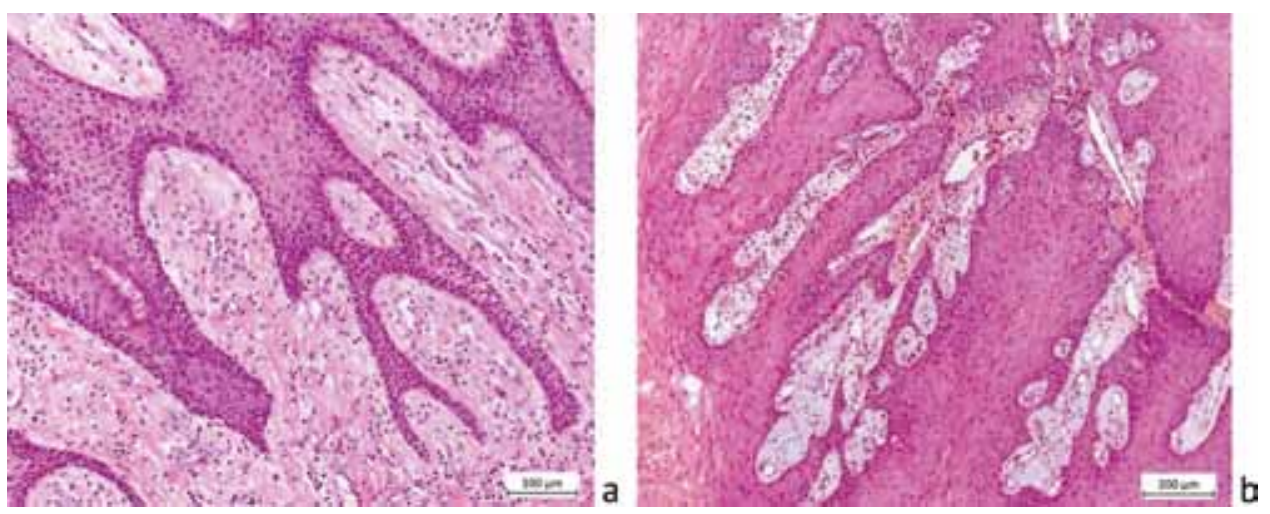

Fig. 1 Epithelial bands growing through the derma with signs of pseudocarcinomous hyperplasia (a). More aggressive proliferation of the epithelial outgrowth is observed with epithelial cells invading the derma $(\boldsymbol{b})$, presence of inflammatory infiltration $(\boldsymbol{a}, \boldsymbol{b})$. Stained with hematoxylin and eosin. Magnification $\times 250(\boldsymbol{a}), \times 125(\boldsymbol{b})$
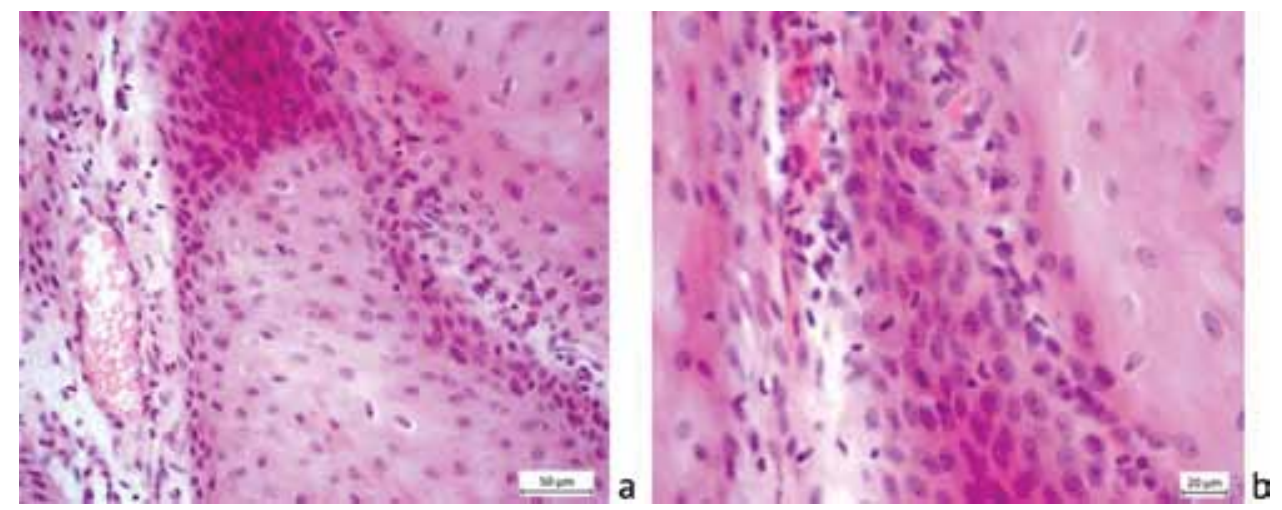

Fig. 2 Mytotic activity, moderately atypical epithelial cells. Stained with hematoxylin and eosin. Magnification $\times 500(\boldsymbol{a})$, $\times 187.5(\boldsymbol{b})$
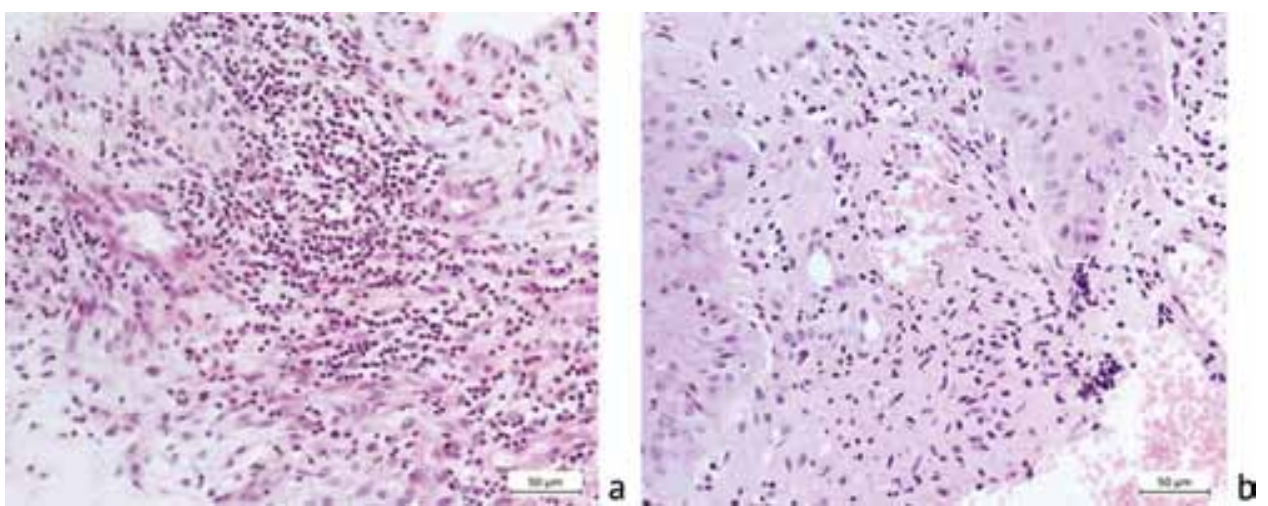

Fig. 3 Focal lymphohistiocytic infiltration and epithelial proliferation $(\boldsymbol{a})$. Diffuse lymphohistiocytic infiltration, clusters of pseudomycelium structures of mycotic bacteria, invasion of epithelial bands $(\boldsymbol{b})$. Stained with hematoxylin and eosin. Magnification $\times 500$

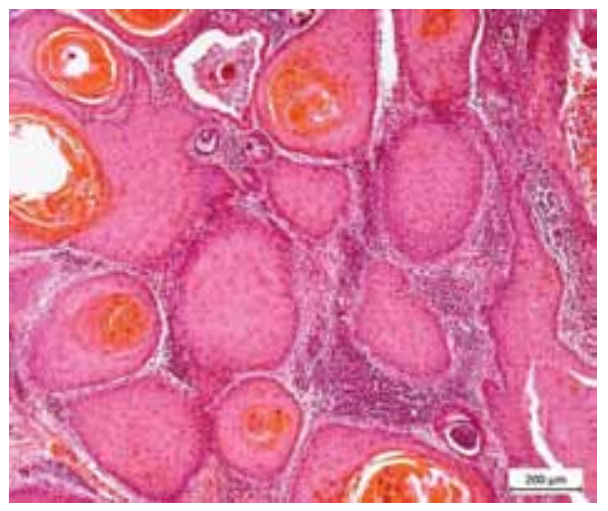

Fig. 4 Formation of multiple corneous pearls, evident infiltration. Stained with hematoxylin and eosin. Magnification $\times 125$ 

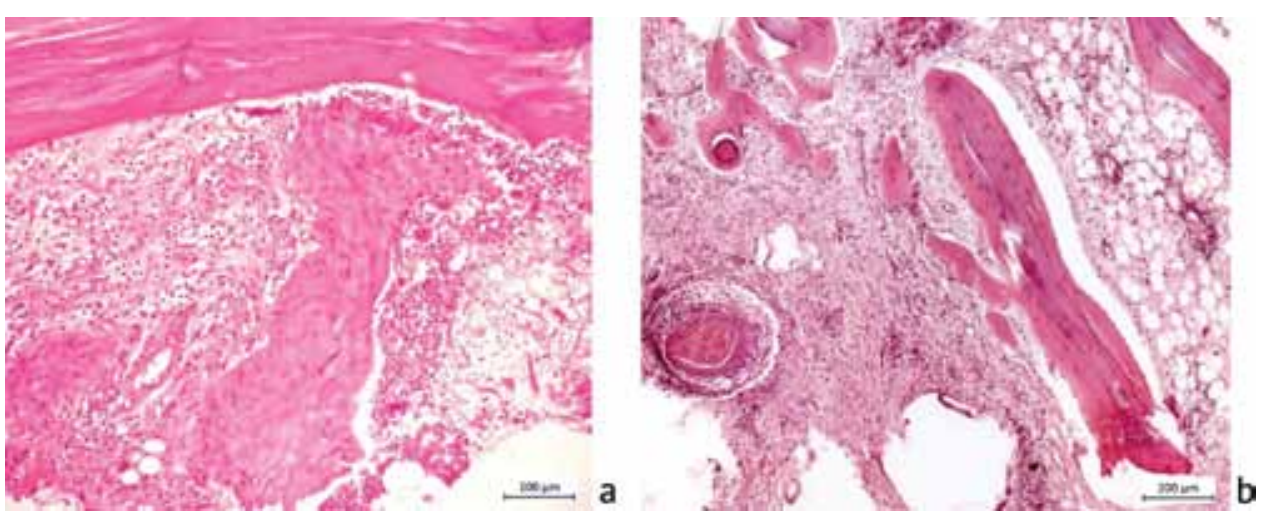

Fig. 5 Neoplastic epithelium invading through the bone tissue without keratosis $(\boldsymbol{a})$ and with keratosis $(\boldsymbol{b})$. Stained with hematoxylin and eosin. Magnification $\times 250(\boldsymbol{a}), \times 125(\boldsymbol{b})$

\section{DISCUSSION}

Squamous cell carcinoma is a rare complication of chronic osteomyelitis and major diagnostic criteria of skin malignancy are critical in long-standing bone infection. Analysis of medical records showed long term condition of chronic osteomyelitis of the foot and ankle with recurrent exacerbations averaged to $36 \pm 6.42$ years.

Physical examination showed skin hyperemia and scars on the involved limb, continuously ( $3 \pm 1.82$ years on average )discharging wounds with an average area of $143.3 \pm 99.4 \mathrm{~cm}^{2}$, malodor and abundant serohemorrhagic exudate. The wound bottom appeared to be lined with loose fibrinous granulations that could be easily injured having elevated cater-shaped hyperemic edges. Hemodynamic changes were detected in the involved limbs by Doppler ultrasonography.

Radiographs demonstrated general radiological findings in addition to individual features of the patients. Bone structure appeared to be heterogeneous, resorptive areas being irregularly outlined, pathological periosteal growth observed alongside with mild radiopaque scarry soft tissues and enhanced odd-shaped clusters in all cases.

Pathomorphology of biopsy samples exhibited pseudocarcinomous hyperplasia that might cause squamous cell carcinoma at a long term. Highly differentiated squamous cell carcinoma was observed in all cases. It should be noted that the above findings were not typical for chronic osteomyelitis. Persistent sinus tracts, growing granulations lining up the bottom of the wound in extensive defects being resistant to mechanical injury are some of the most commonly reported clinical symptoms of chronic osteomyelitis of the foot and ankle [1, 2, 3]. Radiographs showed sharply delineated bone destruction, moderate periosteal reaction and absence of radiopaque pathological ossifications in the soft tissues. Morphological assessment indicated to the absence of neoplastic growth. Therefore, our findings allowed for identification of diagnostic critaria for neoplastic disease associated with pseudocarcinomatous hyperplasia in chronic osteomyelitis.

\section{CONCLUSION}

Diagnosis of neoplastic process as a complication of chronic osteomyelitis with draining sinus can be difficult in the settings of primary or secondary care hospitals where access to highly technical screening instruments is limited. Pseudocarcinomatous hyperplasia can finally transform into squamous cell carcinoma in chronic osteomyelitis with draining sinus or ulceration. Timely recognition and continuous clinical and morphological monitoring are essential for the condition. Squamous cell carcinoma is more characterized as a well differentiated pathology.

\section{REFERENCES}

1. Bobrov M.I., Mitrofanov V.N., Zhivtsov O.P., Nekaeva E.S., Kareva O.V., comp. Lechenie bolnykh pri svishchevom khronicheskom osteomielite (khronicheskii osteomielit s drenirovannym sinusom): nats. klin. rekomendatsii [Treatment of patients for fistulous chronic osteomyelitis (chronic osteomyelitis with drained fistula)]. FGBU "Nizhegorodskii NII Travmatologii i Ortopedii" 
Minzdrava RF, N. Novgorod, 2013. (in Russian) Avalable at: http://www.vossta.ru/federalenoe-gosudarstvennoe-byudjetnoe-v9. html (accessed 05.06.2019).

2. McGrory J.E., Pritchard D.J., Unni K.K., Ilstrup D., Rowland C.M. Malignant lesions arising in chronic osteomyelitis. Clin. Orthop. Relat. Res., 1999, no. 362, pp. 181-189. DOI: 10.1097/00003086-199905000-00027.

3. Lazzarini L., Mader J.T., Calhoun J.H. Osteomyelitis in long bones. J. Bone Joint Surg. Am., 2004, vol. 86, no. 10, pp. $2305-2318$.

4. Gillis L., Lee S. Cancer as a sequel to war wounds. J. Bone Joint Surg. Br., 1951, vol. 33B, no. 2, pp. 167-179.

5. Tkachenko A.N., Noor O.F., Gaykovaya L.B., Korneenkov A.A., Kushnirchuk I.I., Ermakov A.I., Cherkasov A.Yu. Vozmozhnosti differentsialnoi diagnostiki gematogennogo osteomielita i zlokachestvennykh opukholei kostnoi tkani [Possibilities with differential diagnosis of hematogenous osteomyelitis and malignant bone tumors]. Genij Ortopedii, 2018, vol. 24, no. 2, pp. 197-203. (in Russian) DOI: 10.18019/1028-4427-2018-24-2-197-203.

6. Celsus A.C. De Medicina. Ch. XXVI. Spencer W.G., ed. Cambridge, Massachusetts Harvard University Press, 1971.

7. Johnston R.M., Miles J.S. Sarcomas arising from chronic osteomyelitic sinuses. J. Bone Joint Surg. Am., 1973, vol. 55, no. 1, pp. 162-168.

8. Zou J., Guo P., Lv N., Huang D. Lipopolysaccharide-induced tumor necrosis factor- $\alpha$ factor enhances inflammation and is associated with cancer (Review). Mol. Med. Rep., 2015, vol. 12, no. 5, pp. 6399-6404. DOI: 10.3892/mmr.2015.4243.

9. Moura D.L., Ferreira R., Garruço A. Malignant transformation in chronic osteomyelitis. Rev. Bras. Ortop., 2017, vol. 52, no. 2, pp. 141-147. DOI: 10.1016/j.rboe.2017.03.005.

10.Li Q., Cui H., Dong J., He Y., Zhou D., Zhang P., Liu P. Squamous cell carcinoma resulting from chronic osteomyelitis: a retrospective study of 8 cases. Int. J. Clin. Exp. Pathol., 2015, vol. 8, no. 9, pp. 10178-10184.

11.Rauh M.A., Duquin T.R., McGrath B.E., Mindell E.R. Spread of squamous cell carcinoma from the thumb to the small finger via the flexor tendon sheaths. J. Hand Surg. Am., 2009, vol. 34, no. 9, pp. 1709-1713. DOI: 10.1016/j.jhsa.2009.06.012.

12.Panteli M., Puttaswamaiah R., Lowenberg D.W., Giannoudis P.V. Malignant transformation in chronic osteomyelitis: recognition and principles of management. J. Am. Acad. Orthop. Surg., 2014, vol. 22, no. 9, pp. 586-594. DOI: 10.5435/JAAOS-22-09-586.

Received: 22.05.2019

\section{Information about the authors:}

1. Nikolai M. Kliushin, M.D., Ph.D., Russian Ilizarov Scientific Center for Restorative Traumatology and Orthopaedics, Kurgan, Russian Federation, Email: klyushin_nikolay@mail.ru

2. Anatolii S. Sudnitsyn, M.D., Ph.D., Russian Ilizarov Scientific Center for Restorative Traumatology and Orthopaedics, Kurgan, Russian Federation, Email: anatol_anatol@mail.ru

3. Nikolai S. Migalkin,

Russian Ilizarov Scientific Center for Restorative Traumatology and Orthopaedics, Kurgan, Russian Federation

4. Tatyana A. Stupina, Ph.D. of Biological Sciences,

Russian Ilizarov Scientific Center for Restorative Traumatology and Orthopaedics, Kurgan, Russian Federation, Email: StupinaSTA@mail.ru

5. Nikita R. Suvorov,

Tikhvin Interdistrict Hospital named after A.F. Kalmykova", Leningrad Region, Tikhvin, Russian Federation 